'Servicio de Neurología, Hospital Barros Luco, Santiago, Chile. ${ }^{2}$ Facultad de Ciencias Médicas, Universidad de Santiago de Chile. ${ }^{3}$ Facultad de Medicina, Universidad de Chile.

Recibido el 15 de julio de 2014, aceptado el 8 junio de 2015.

Correspondencia a: Dr. Luis Lay-Son Rivas Avda. JM Carrera 3204, San Miguel, Santiago, Chile. Teléfono: 56-2-25763337 luis.layson@usach.cl

\section{Latencia diagnóstica en la enfermedad de Parkinson. Estudio en 200 pacientes de novo en un hospital público de Chile}

\author{
LUIS LAY-SON ${ }^{1,2}$, CLAUDIO ELOIZA ${ }^{1,3}$, OSVALDO TRUJILLO-GODOY $^{1,3}$
Delay in the diagnosis of Parkinson's disease in a Chilean public hospital

\begin{abstract}
Background: Early diagnosis is fundamental in patients with Parkinson's disease (PD) to improve their quality of life. Aim: To determine the latency in the diagnosis of Parkinson's disease $(P D)$ after the onset of motor symptoms. Patients and Methods: Prospective study carried out during 16 months in a public hospital. Two hundred newly diagnosed patients aged 41 to 90 years (50\% women), were included and analyzed. Results: The lapse between the first symptom-more commonly tremor-and the diagnosis made by a neurologist ranged from 1 to 84 months (19.1 \pm 13.8$)$. In $39 \%$ of patients, it was done in the first year, in $26 \%$ during the second year and in $35 \%$ of patients, it took more than two years. The referral by a general practitioner had a delay ranging from 1 to 36 months. Sixty nine and 95\% of patients were evaluated within the first 6 months after referral if they came from primary care or the same hospital, respectively. Twenty six percent of patients were classified in stages III to V of Hoehn \& Yahr's staging and the Parkinson's Disease Rating Scale motor examination ranged from 5 to 81 points, mean 24 ( \pm 12.8 ). Conclusions: The diagnosis of PD has a delay in a public hospital that could be influenced by the referral system.
\end{abstract}

(Rev Med Chile 2015; 143: 870-873)

Key words: Delayed diagnosis; Delivery of Health care; Parkinson disease.

\section{L} a enfermedad de Parkinson (EP) es un trastorno degenerativo, sistémico, progresivo que afecta prioritariamente las funciones motoras, cognitivas, mentales y conductuales y va generando, gradual e inexorablemente, una discapacidad laboral y luego un deterioro en las actividades sociales y personales a lo largo de su evolución. Esta enfermedad, no obstante su conocimiento antiguo y universal, presenta un retardo en ser reconocida y más aun se encuentra sub-diagnosticada ${ }^{1,2}$. El diagnóstico precoz constituye una herramienta fundamental para mejorar la calidad de vida de los pacientes portadores de la enfermedad, en especial aquellos pertenecientes a los grupos etarios más jóvenes, teniendo en cuenta que se encuentran activos laboralmente y además presentan una peor evolución. El objetivo del presente trabajo fue determinar la latencia desde la detección del primer síntoma motor por parte del paciente o familiar y el momento de la confirmación diagnóstica en nuestro centro de atención secundaria.

\section{Pacientes y Métodos}

Se trata de un estudio de tipo observacional y descriptivo, realizado de manera prospectiva entre julio de 2010 y octubre de 2011 en el Centro de Diagnóstico y Tratamiento de nuestro hospital, ubicado en Santiago de Chile, con una población asignada de aproximadamente 1.200.000 habitantes. Un grupo consecutivo de 200 pacientes de novo examinados por los autores, y que cumplían 
con los criterios de la EP idiopática, fueron inclui$\operatorname{dos}^{3}$. Aquellos casos que podían corresponder a parkinsonismo secundario a patología vascular o farmacológica o con diagnóstico dudoso fueron descartados. El análisis incluyó los aspectos demográficos generales, historia familiar, temblor esencial previo y el lado corporal inicialmente comprometido. En cuanto a la evaluación específica, se usaron las clásicas escalas de Hoehn y Yahr (HY) para definir el estadio de progresión de la enfermedad y la Unified Parkinson's Disease Rating Scale Motor Examination (UPDRS-ME) para determinar el puntaje de los síntomas motores. Los pacientes fueron clasificados en dos grupos clínicos como tembloroso o acinético-rígido mediante el uso de los ítems 20, 21, 22, 23, 24, 25 y 26 de esta última escala. Mediante el método Mann-Whitney y Kruskal-Wallis se analizaron las variables clínicas y demográficas en la búsqueda de posibles factores determinantes de una mayor latencia diagnóstica. Es importante establecer que el presente trabajo no implicó una intervención diagnóstica ni terapéutica especial ni uso de información personalizada, no obstante lo anterior, nuestra investigación fue aprobada por el comité de ética institucional.

\section{Resultados}

El estudio se focalizó en analizar la variable en estudio en el grupo diagnosticado, sin añadir otros aspectos como la referencia con diagnóstico erróneo $\mathrm{u}$ otros indicadores como la incidencia de la enfermedad. Las características generales y clínicas se detallan a continuación (Tabla 1). La distribución de los pacientes según género fue
1:1, con un rango etario de 41 a 90 años y una mediana de $73( \pm 9,8)$. No hubo diferencias en cuanto al promedio de edad por sexo. El 61\% de los pacientes era mayor de 70 años con $18 \%$ de octogenarios. El 66,5\% de los pacientes tenía escolaridad nula o básica. En relación al domicilio, la mayoría residía en zona urbana (81\%). De los 35 pacientes que presentaban una historia positiva de EP en un familiar directo, $40 \%$ de los casos correspondía a un hermano (a). Por otro lado, solamente 9 pacientes tenían el antecedente de temblor esencial (TE). Un grupo mayoritario de pacientes $(78,5 \%)$ fueron referidos por médicos de atención primaria (AP) y el resto fue derivado por otros especialistas de atención secundaria (AS) del mismo hospital. La generalidad de los pacientes $(88 \%)$ fueron referidos con un diagnóstico presuntivo de EP o TE, siendo la demencia el segundo diagnóstico más frecuente. La evaluación neurológica inicial mostró un franco predominio del cuadro clínico tembloroso $(74,5 \%)$ por sobre el tipo aquinético-rígido. En cuanto al hemicuerpo en el cual debutó la EP se obtuvo la siguiente información, $44,5 \%$ a derecha, $43,5 \%$ a izquierda y $12 \%$ restante no logró precisar el lado. Los pacientes se distribuyeron en todos los estadios HY, pero resaltaba el hecho que $25,5 \%$ de ellos fueron clasificados entre los estadios III-V, es decir, tenían una franca alteración de los reflejos posturales. Los síntomas motores de acuerdo a la escala UPDRS-ME presentaban una mediana de $24( \pm 12,8)$ puntos con un rango de 5-81, destacando que más de la mitad (64\%) tenían un puntaje superior a 20 puntos. No hubo diferencias en el puntaje de la UPDRS-ME al comparar mujeres vs hombres, ni al compararlo por intervalos de edad.

Tabla 1. Características generales de 200 pacientes parkinsonianos al momento del diagnóstico

\begin{tabular}{|lll|}
\hline Edad & $73( \pm 9,8)$ años & Rango $41-90$ \\
\hline Sexo & Mujer $50 \%$ & Hombre $50 \%$ \\
\hline Escolaridad & $<8$ años $66,5 \%$ & $>8$ años $33,5 \%$ \\
\hline Residencia & Urbana $81 \%$ & Rural $19 \%$ \\
\hline Historia familiar EP & No $82,5 \%$ & Sí $17,5 \%$ \\
\hline Cuadro clínico & Tembloroso $74,5 \%$ & Aquinético-rígido $25,5 \%$ \\
\hline Escala Hoehn-Yahr & Estadio I-II: $74,5 \%$ & Estadio III-V 25,5\% \\
\hline Escala UPDRS-III & $24( \pm 12,8)$ puntos & Rango 5-81 \\
Lugar de referencia & Atención primaria $78,5 \%$ & Atención secundaria $21,5 \%$ \\
\hline
\end{tabular}


Tabla 2. Latencia diagnóstica en 200 pacientes con EP

\begin{tabular}{|c|c|c|c|}
\hline \multicolumn{4}{|c|}{ Mediana 19,1 $( \pm 13,8)$ rango $1-84$ meses } \\
\hline$<6$ meses & 34 & pacientes & $17 \%$ \\
\hline 6-12 meses & 45 & & $22,5 \%$ \\
\hline 13-24 meses & 51 & & $25,5 \%$ \\
\hline 25-36 meses & 42 & & $21 \%$ \\
\hline$>37$ meses & 28 & & $14 \%$ \\
\hline
\end{tabular}

La latencia diagnóstica entre el primer síntoma detectado por el paciente o un familiar -generalmente el temblor- y la confirmación diagnóstica llevada a cabo por uno de los neurólogos del equipo varió entre 1 y 84 meses con una mediana $19,1( \pm 13,8)$. Solamente en $39,5 \%$ de los casos hubo un diagnóstico realizado dentro de los primeros 12 meses. En cambio, en 35\% hubo una demora mayor a dos años, de los cuales 14\% fueron diagnosticados sobre los 3 años (Tabla 2). No se encontró estadísticamente significativo que los siguientes factores desempeñaran un rol determinante: edad $(<60,61-70,>71$ años); género; domicilio (urbano vs rural); escolaridad ( $<8$ años vs $>8$ años); lugar de derivación (AP vs AS); tipo de cuadro clínico (tembloroso vs aquinético-rígido); puntaje en el UPDRS-ME $(<20$, 21-40, > 40 puntos).

La latencia en la referencia definida por el tiempo que transcurrió desde el instante que el paciente fuera derivado por otro colega hasta recibir la atención del especialista varió entre 1 y 36 meses incluyendo el grupo en su totalidad. El sub-grupo referido desde la AP fue analizado de acuerdo a la residencia, no encontrándose -contrario a lo esperado- que la ruralidad constituyese un factor asociado a una mayor latencia. En cambio, hubo una diferencia significativa entre los pacientes referidos desde la AP y los pacientes derivados desde AS. El 95\% de los pacientes referidos desde el mismo complejo hospitalario fueron atendidos dentro de los siguientes 6 meses vs $68,5 \%$ referido desde AP.

\section{Discusión}

El proceso diagnóstico en la EP depende de la detección de los síntomas motores por parte del paciente o familiar, del médico de atención primaria y finalmente del especialista. Al revisar la literatura sólo encontramos tres trabajos que compartían un grado de similitud con el nuestro $^{4-6}$. Al comparar los datos generales hallamos que al momento del diagnóstico la edad en nuestro grupo fue ligeramente mayor que el promedio de 67 años obtenido en dos informes previos ${ }^{4,5}$. En relación al aspecto clínico, el grupo de 131 pacientes de Post et al. presentaba un compromiso motor menor al nuestro, con $88 \%$ distribuido en los estadios I-II de HY, y una mediana de 17,6 (5-40) puntos en la UPDRS-ME ${ }^{4}$. En el grupo de Breen et al, los 239 pacientes se ubicaban en los estadios I-II de HY pero con un puntaje de 31 (23-38) en la UPDRS-ME5 . La latencia diagnóstica en nuestro trabajo fue muy similar al resultado de Post et al que mostró una latencia de $20(4,7-$ 83,9) meses $^{4}$. El trabajo de Han et al, realizado en Beijing a través de una encuesta, demostró que los pacientes consultaron al médico general en promedio 6,7 meses después del inicio de los síntomas y que la mitad de ellos tardaron más de un año en ser confirmados con la EP con el especialista ${ }^{6}$. El trabajo de Breen et al, realizado en Inglaterra en base a datos obtenidos de un estudio epidemiológico comunitario, informó una latencia mediana de 11 (0-100) meses en la consulta al médico general y una latencia mediana de 1 (0-53) meses entre esta consulta y el diagnóstico por el neurólogo ${ }^{5}$. En el mismo trabajo, tras evaluar la participación de múltiples variables demográficas y clínicas, se concluyó que la condición de varón y el cuadro clínico acinético-rígido en forma separada eran los únicos factores que determinaban un incremento en la latencia diagnóstica. Por el contrario, en otro trabajo se concluyó que la condición de mujer resultaba en una mayor latencia diagnóstica ${ }^{7}$. En cambio, en nuestro análisis no hallamos que el género ni ningún otro factor fuese determinante de un mayor retardo en el diagnóstico de la EP. En Chile, la mayoría de los pacientes se atienden en el sistema público de salud y, en general, requieren una interconsulta generada por el médico de AP para ser evaluados por un especialista de AS. De este modo, resulta fundamental la sospecha diagnóstica de la EP por parte de los médicos de AP. En sendos trabajos foráneos se constató que un porcentaje de pacientes fueron referidos al especialista luego de múltiples consultas $^{6,8}$. La referencia desde la AP constituye un elemento responsable de incrementar la latencia 
diagnóstica, en contraste con la resolución más expedita de la interconsulta proveniente desde la AS. En forma ideal, la intervención terapéutica del paciente con EP debería ocurrir en el estadio I de la escala de HY o un puntaje menor a los 10 puntos en la escala UPDRS-ME. Se debe optimizar la detección de la EP en la comunidad en búsqueda de los síntomas motores que son más precoces $\mathrm{y}$ evidentes, aunque hoy existe una tendencia a privilegiar la pesquisa y análisis de los síntomas no motores, pero a excepción de la depresión, estos síntomas son más ostensibles cuando el cuadro mórbido está en su máxima expresión ${ }^{9}$. Para tal efecto, hemos diseñado una guía de síntomas y signos motores y no motores que ayuden a los pacientes o parientes a pesquisar de manera más precoz la presencia de esta enfermedad y así disminuir la brecha que separa al paciente del neurólogo (Anexo 1).

\section{Conclusión}

El diagnóstico precoz de la EP tiene un pilar fundamental que es la educación, la cual debe estar dirigida a la población general y a los médicos de atención primaria para una pronta evaluación por el especialista. Además, es perentorio agilizar el procesamiento de las interconsultas externas que es un asunto netamente administrativo manejado por la red asistencial.

\section{Referencias}

1. Schoenberg BS, Anderson DW, Haerer AF. Prevalence of Parkinson's disease in the biracial population of Copiah County, Mississippi. Neurology 1985; 35 (5): 841-5.

2. Hoglinger GU, Rissling I, Metz A, Ries V, Heinermann A, Prinz H, et al. Recognition of Early Parkinsonism in the Community. Movement Disorders 2004; 19 (5): 505-12.

3. Gibb WR, Lees AJ. The relevance of the Lewy body to the pathogenesis of idiopathic Parkinson's disease. J Neurol Neurosurg Psychiatry 1988; 51 (6): 745-52.

\section{Anexo 1. Síntomas o signos sugerentes de la Enfermedad de Parkinson}
1. Temblor de mano o cabeza
2. Pesadez de extremidades inferiores
3. Lentitud en un hemicuerpo
4. Rigidez de hombro o mano
5. Alteración del equilibrio o caídas
6. Marcha lenta o pasos cortos
7. Voz apagada o dificultosa
8. Dificultad para usar herramientas o escribir
9. Falta de ánimo o gratificación
10. Pérdida del olfato
11. Pérdida de memoria
12. Constipación pertinaz
13. Sueño inquieto
14. Disminución en la autovalencia no acorde a la edad

4. Post B, Speelman JD, de Haan RJ. Clinical heterogeneity in newly diagnosed Parkinson's disease. J Neurol 2008; 255 (5): 716-22.

5. Breen DP, Evans JR, Farrell K, Brayne C, Barker RA. Determinants delayed diagnosis in Parkinson's disease. J Neurol 2013; 260 (8): 1978-81.

6. Han Y, Zhang XH, Chen Tong, Wang Z, Sun H. Survey of diagnosis and therapy in the Parkinson's disease. Chinese Journal of Health Care and Medicine 2008; 10 (1): 18-20.

7. Saunders-Pullman R, Wang C, Stanley K, Bressman SB. Diagnosis and referral delay in women with Parkinson's disease. Gend Med 2011; 8 (3): 209-17.

8. Gonera EG, van't Hof M, Berger HJ, van Weel C, Horstink MW. Symptoms and duration of the prodromal phase in Parkinson's disease. Mov Disord 1997; 12 (6): 871-6.

9. Barone P, Antonini A, Colosimo C, Marconi R, Morgante L, Avarello TP. The PRIAMO study: A multicenter assessment of nonmotor symptoms and their impact on qualityof life in Parkinson's disease. Movement Disorders 2009; 24 (11): 1641-9. 